\title{
Chorioamniotic Separation Found on Obstetric Ultrasound and Perinatal Outcome
}

\author{
Carolina Bibbo, MD ${ }^{1}$ Sarah E. Little, MD, MPH ${ }^{1}$ Jad Bsat, BA ${ }^{1}$ Kris Ann Botka, BA, RDMS ${ }^{2}$ \\ Carol B. Benson, $\mathrm{MD}^{2}$ Julian N. Robinson, $\mathrm{MD}^{1}$ \\ ${ }^{1}$ Division of Maternal Fetal Medicine, Department of Obstetrics and \\ Address for correspondence Carolina Bibbo, MD, Department of \\ Gynecology, Brigham and Women's Hospital, Boston, Massachusetts \\ 2 Department of Radiology, Brigham and Women's Hospital, \\ Boston, Massachusetts \\ Obstetrics and Gynecology, Brigham and Women's Hospital, 75 Francis \\ Street, Boston, MA 02115 (e-mail: cbibbo@partners.org).
}

Am J Perinatol Rep 2016;6:e337-e343.

\begin{abstract}
Keywords

- chorioamniotic separation

- preterm delivery rate

- stillbirth

- aneuploidy

Objective This study aims to evaluate pregnancy outcomes in patients with spontaneous and iatrogenic chorioamniotic separation diagnosed by ultrasound after 17 weeks.

Methods This is a retrospective cohort study of women with a singleton pregnancy who were diagnosed with chorioamniotic separation $(n=106)$ after 17 weeks' gestation from January 2000 to January 2013. Patients with chorioamniotic separation were compared with a group of patients who had obstetric ultrasounds without a diagnosis of chorioamniotic separation. Those without chorioamniotic separation were matched (1:1) on gestational age on the date of the ultrasound ( \pm 2 weeks) $(n=106)$. The primary outcome was preterm delivery ( $<37$ weeks). Secondary outcomes included intrauterine growth restriction, stillbirth, and neonatal morbidity.

Results The rate of preterm delivery was significantly higher for those with chorioamniotic separation than for those without ( 57.5 vs. $17.1 \%, p<0.0001$ ). There were no significant differences in the rate of aneuploidy, intrauterine growth restriction, stillbirth, or neonatal demise. The rate of stillbirth was significantly higher among those with chorioamniotic separation diagnosed before 24 weeks as compared with those diagnosed after 24 weeks ( 9.7 vs. $0 \%, p=0.03$ ).

Conclusions Chorioamniotic separation is associated with preterm delivery. If diagnosed before 24 weeks, the rate of stillbirth is significantly higher.
\end{abstract}

The separation of the chorion and amnion before 14 weeks' of gestation is physiologically normal. The amnion and chorion usually fuse between 14 and 16 weeks, and any chorioamniotic separation (CAS) that persists after 16 weeks is uncommon and anomalous. ${ }^{1}$ CAS can occur spontaneously or after an intrauterine intervention such as amniocentesis, fetal blood sampling, or fetal surgery. ${ }^{2,3}$

The vast majority of the reported cases of CAS have been of pregnancies that were exposed to an invasive intrauterine procedure, mainly a genetic amniocentesis or fetal surgery. The literature regarding spontaneous CAS is sparse with only a small number of cases. ${ }^{3,4}$ Prior research has shown that CAS

received

August 14, 2016

accepted

August 18, 2016
DOI http://dx.doi.org/

10.1055/s-0036-1593407. ISSN 2157-6998. seen after 17 weeks is associated with adverse perinatal outcomes, such as amniotic band syndrome, umbilical cord strangulation, and fetal death. ${ }^{3,5}$ CAS has also been associated with fetal aneuploidy, particularly trisomy 21, preterm delivery, premature rupture of membranes, and fetal growth restriction. ${ }^{2,6-8}$ A literature review on CAS by Lewi et al encompassed 47 cases of severe CAS between 1982 and 2004 in which the rate of preterm birth was $40 \%$ and the rate of intrauterine fetal demise or miscarriage was $28 \%{ }^{8}$

The limited case reports on this subject have made it difficult to counsel patients regarding the prognosis of CAS, especially if the separation is spontaneous. Thus, our goal was to study the
Copyright $\odot 2016$ by Thieme Medical Publishers, Inc., 333 Seventh Avenue, New York, NY 10001, USA. Tel: +1(212) 584-4662.
License terms

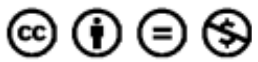


effect of CAS on pregnancy outcome, primarily, preterm delivery. In addition, we sought to evaluate whether the etiology (spontaneous vs. iatrogenic), the timing of diagnosis, and the degree of severity of the CAS impacted outcome.

\section{Methods}

This is a single-center retrospective cohort study of women who were diagnosed with CAS $(n=106)$ beyond 17 weeks' gestation from January 2000 to January 2013. This cohort underwent an ultrasound examination at the Brigham and Women's Hospital. Potential cases of CAS were identified using the hospital's ultrasound database, which is an electronic, searchable database of all obstetric ultrasound reports during the study period. The terms used for the search were "chorioamniotic" or "chorio-amniotic" in the "comments" field. CAS is the separation of the chorion and amnion layers by an anechoic space (-Fig. 1). The exact distance between the two membranes to meet the criteria for CAS has not been clearly established and there is no standardized classification for severity based on the depth of the separation. At our institution, we consider that the depth of the CAS is not an accurate representation of the size of the separation since we believe it depends on its overall shape and on how much of the cavity is involved. When the idea of this study originated, the cases of CAS were retrospectively graded. The cases were classified as mild, moderate, or severe by two senior physicians, one maternal-fetal medicine specialist, and one radiologist. Cases were subjectively graded as mild if the CAS was limited to a quarter of the uterine cavity, moderate if it extended between a quarter and half of the uterine cavity, and severe if it extended for more than half of the uterine cavity. If there were not sufficient images or movie clips to clearly classify the separation, these cases were excluded.
We collected demographic, medical history, and prenatal data through chart review. The primary outcome of the study was preterm delivery (less than 37 weeks' of gestation). Secondary outcomes included: gestational age at the time of delivery, spontaneous preterm delivery, preterm premature rupture of membranes, abruption, chorioamnionitis, fetal intrauterine growth restriction (ultrasound estimated fetal weight below the 10th percentile for gestational age), 5-minute Apgar score less than 7 , neonatal intensive care unit admission, intrauterine fetal demise (death of fetus after 20 weeks and before complete expulsion or extraction from the mother, irrespective of the duration of the pregnancy), neonatal demise (infant death from birth until 28 days of life), and mode of delivery.

Furthermore, we compared maternal and pregnancy characteristics as well as pregnancy outcomes between cases of CAS that occurred spontaneously (spontaneous) and cases that occurred after an invasive intrauterine procedure (iatrogenic). The spontaneous CAS group included patients that did not have an invasive intrauterine procedure during the pregnancy or that had an intrauterine invasive procedure only after the diagnosis of CAS was made. The group of iatrogenic CAS included pregnancies that were diagnosed with a CAS after an invasive intrauterine procedure. In addition, we compared pregnancy outcomes of patients with CAS based on their gestational age at diagnosis (before vs. after 24 weeks), and their degree of severity (mild vs. moderate/severe).

Assuming a national baseline preterm birth rate of $11.4 \%,{ }^{9}$ 33 patients would be needed in each group to have an $80 \%$ power to detect an increase in preterm birth rate to $40 \%{ }^{8}$ with an $\alpha$ error of $5 \%$. Since our case group (CAS group) was large enough to detect this difference, we selected the same number of controls as participants (1:1). The control group was identified from the same electronic ultrasound database. We searched our database for control patients who had had

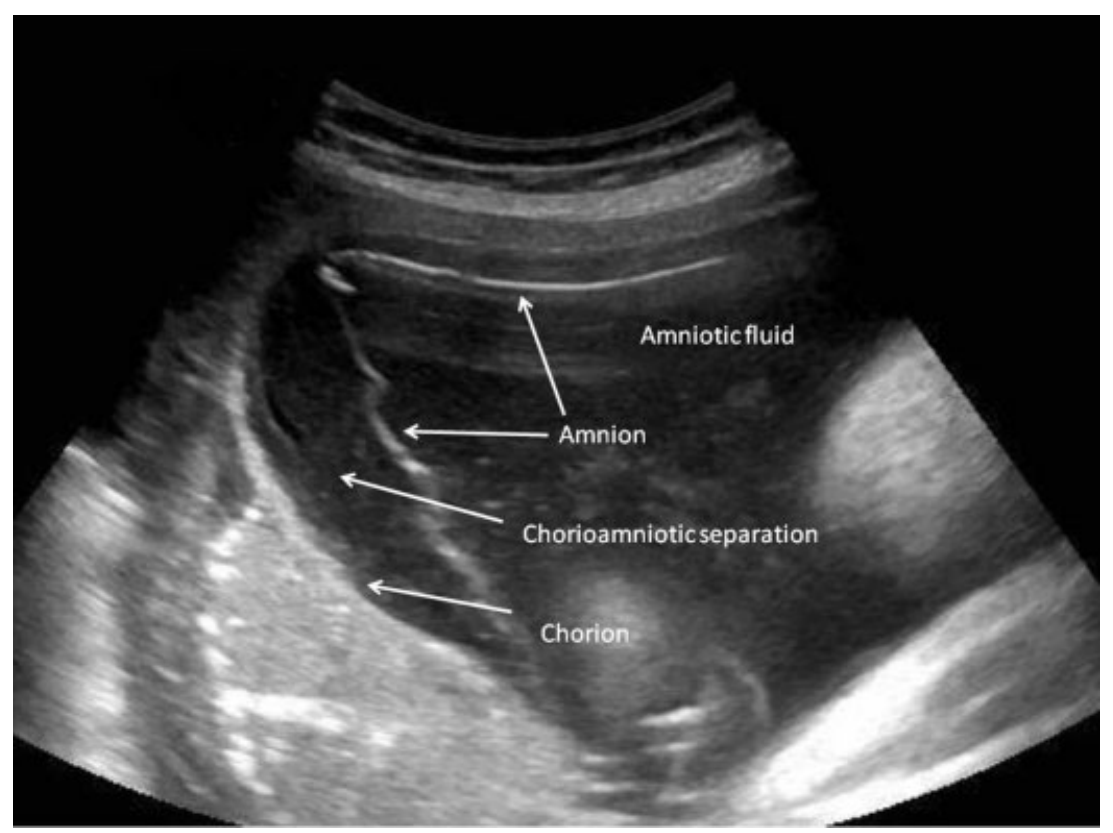

Fig. 1 This figure represents the chorioamniotic separation diagnosed by ultrasound evaluation at 39 weeks. The indication for the ultrasound evaluation was to obtain an estimated fetal weight. The arrows point at the amniotic membrane that has separated from the chorion. 
an ultrasound on the same date/year as those patients who were diagnosed with a CAS, but who were not diagnosed with a CAS. We matched for singleton gestation and gestational age at the time of ultrasound ( \pm 2 weeks' gestation). The prior and subsequent ultrasound reports of the control participants were reviewed to ensure that there was no mention of CAS, and the ultrasound images were also reviewed to ensure that the diagnosis was not missed on the available captured images.

Univariate statistical analysis was performed using chisquare or Fisher exact tests when appropriate for categorical variables and Wilcoxon rank sum testing for continuous variables. Multivariate logistic regression was used to test the independent association of CAS and preterm delivery. The $p$ values $\leq 0.05$ were considered statistically significant.

In addition, we performed a sensitivity analysis where we only included a subgroup of patients who did not have risk factors for preterm delivery. In this analysis, we excluded patients whose indications for ultrasound were associated with risk of preterm labor (ultrasound indications: symptoms of pain, vaginal bleeding, leakage of fluid, preterm labor; cervical length measurement). We also excluded patients with a history of subchorionic hematoma, history of prior spontaneous preterm birth, and all patients that had undergone any invasive intrauterine procedure. This subgroup analysis excluded 45 controls and 81 cases from the original cohort; the sensitivity analysis contained 61 controls and 25 cases (23 cases with outcome data). The primary outcome, preterm delivery, was compared between cases and controls in this subset population.

Study data were collected and managed using REDCap electronic data capture tools hosted at Brigham and Women's Hospital and Harvard Medical School. ${ }^{10}$ SAS 9.3 (SAS Institute, Inc., Cary, NC) software was used for statistical analyses. The Partners Healthcare Institutional Review Board approved this project on November 16, 2012 (protocol number: 2012P002128).

\section{Results}

Between January 2000 and January 2013, the ultrasound database search yielded 155 cases where there might have been a CAS after 17 weeks' gestation. Upon review of the images, 49 cases were excluded, as the images did not meet the criteria to classify CAS. A total of 106 singleton pregnancies were included in the CAS group and 106 singleton pregnancies were included in the control group. There was no pregnancy outcome information for nine patients in the CAS group. Either these patients were lost to follow-up or they delivered at an outside institution. There were a total of four elective terminations of pregnancy that were excluded from part of the pregnancy outcome analysis (but not excluded from pregnancy characteristics and incidence of aneuploidy analyses). There was one termination in the control group for a fetus with trisomy 21 and three terminations in the CAS group, two with trisomy 18 and one with a fetus with a bladder outlet obstruction. With these exclusions, our final sample size for analyzing pregnancy outcomes was 94 cases and 105 controls.
There were statistically significant differences between the CAS and control groups in the rate of amniocentesis, vaginal bleeding, subchorionic hematoma, indication for ultrasound evaluation, and oligohydramnios and polyhydramnios at the time of the initial diagnosis of CAS or the respective ultrasound for the control group ( - Table 1 ). The rate of preterm delivery, our primary outcome, was significantly different between the two cohorts: $57.5 \%$ for the CAS group compared with $17.1 \%$ for the control group $(p<0.0001$; - Table 2$)$. CAS was highly associated with a greater than fourfold increased risk of spontaneous preterm delivery (39.4 vs. 9.5\%, $p<0.0001$; - Table 2). In addition, there were significant differences between the two cohorts in the rate of chorioamnionitis, placental abruption, birth weight, 5-minute Apgar score of less than 7, and the rate of neonatal intensive care unit admission (-Table 2 ). There were no significant differences in the rate of intrauterine growth restriction, stillbirth, neonatal demise, or mode of delivery (-Table 2).

In the CAS group, the median gestational age at diagnosis was 29.7 weeks (interquartile range [IQR], 19.1-35.1 weeks). The median time from diagnosis to rupture of membranes in the overall CAS group was 1.6 weeks (IQR, 0.4-5.0 weeks) and the median time from diagnosis to delivery was 1.25 weeks (IQR, 0.3-8.7 weeks). Regarding the cases of CAS, there were 74 cases $(69.8 \%)$ of spontaneous CAS and 32 cases $(30.2 \%)$ of iatrogenic CAS. The spontaneous CAS group included 15 patients that had an intrauterine invasive procedure after the diagnosis of CAS was made: 13 had a genetic amniocentesis, 1 had an amniocentesis to rule out chorioamnionitis, and another one had an indigo carmine test to rule out rupture of membranes. The group of iatrogenic CAS included a total of 32 patients: 27 had an amniocentesis, 4 had chorionic villous sampling, and 1 had a percutaneous umbilical cord blood sampling. The median gestational age at diagnosis was not different between the spontaneous and iatrogenic groups (30 weeks [IQR, 19.2-36.3 weeks] vs. 26.8 weeks [IQR, 19.3-34.6 weeks], $p=0.48$ ). The persistence of CAS throughout subsequent ultrasounds was also not different between the two cohorts ( 70.3 vs. $65.5 \%, p=0.57$ ).

Pregnancy outcomes were stratified based on whether the CAS occurred spontaneously or after a procedure, the timing of diagnosis, and degree of severity of the CAS (-Table $\mathbf{3}$ ). There were no significant differences in pregnancy outcomes between the spontaneous and iatrogenic cases; however, there was a trend toward more cases of intrauterine growth restriction with spontaneous CAS than with iatrogenic CAS ( 12.5 vs. $0 \%, p=0.05$ ). There were no significant differences in pregnancy outcomes between mild and moderate/severe group, but there was a trend toward more cases of stillbirth in the moderate/severe group (7.7 vs. $0 \%, p=0.06$ ). When comparing pregnancy outcomes based on the timing of the diagnosis, there was no difference in the overall preterm delivery rate, but the rate of delivery prior 28 weeks was higher in the group diagnosed before 24 weeks ( 25.8 vs. 3.2\%, $p=0.002$; - Table 3 ). The rate of stillbirth was also significantly higher in the group diagnosed before 24 weeks ( 9.7 vs. $0 \%, p=0.03$ ). For those pregnancies diagnosed before 24 weeks, the median time from diagnosis to rupture of 
Table 1 Maternal and pregnancy characteristics of the control and case groups

\begin{tabular}{|l|l|l|l|}
\hline Characteristic & $\begin{array}{l}\text { Case group } \\
(\boldsymbol{n}=106)\end{array}$ & $\begin{array}{l}\text { Control group } \\
(\boldsymbol{n}=\mathbf{1 0 6})\end{array}$ & $\boldsymbol{p}$ Value \\
\hline Age (y) & $33.5(29-37)$ & $32.5(28-37)$ & 0.33 \\
\hline Advanced maternal age (y) & $47(44.3)$ & $37(34.9)$ & 0.16 \\
\hline Race & & & 0.68 \\
\hline White & $75(70.8)$ & $70(66.0)$ & \\
\hline Black & $11(10.4)$ & $16(15.1)$ & \\
\hline Hispanic & $8(7.6)$ & $10(9.4)$ & \\
\hline Asian & $12(11.3)$ & $10(9.4)$ & \\
\hline Nulliparous & $49(46.2)$ & $46(43.4)$ & 0.68 \\
\hline Conceived by in vitro fertilization & $13(12.6)$ & $10(9.4)$ & 0.51 \\
\hline History of spontaneous preterm birth & $9(8.5)$ & $13(12.3)$ & 0.37 \\
\hline Intrauterine procedure & & \\
\hline Chorionic villous sampling & $4(3.8)$ & $4(3.8)$ & 1.00 \\
\hline Amniocentesis & $42(40.0)$ & $21(19.8)$ & $\mathbf{0 . 0 0 1}$ \\
\hline Aneuploidy & $6(6.2)$ & $2(1.9)$ & 0.16 \\
\hline Vaginal bleeding & $24(22.4)$ & $9(8.5)$ & $\mathbf{0 . 0 0 5}$ \\
\hline Subchorionic hematoma & $18(17.0)$ & $1(0.9)$ & $<\mathbf{0 . 0 0 1}$ \\
\hline Indication for ultrasound & & & $<0.001$ \\
\hline Symptoms: Vaginal bleeding, pain, leakage of fluid, preterm labor & $29(27.4)$ & $6(5.7)$ & \\
\hline Cervical length & $4(3.8)$ & $11(10.4)$ & \\
\hline Scheduled survey & $16(15.1)$ & $26(24.5)$ & \\
\hline Abnormal anatomy follow up & $12(11.3)$ & 0 & $1(0.9)$ \\
\hline Abnormal finding on ultrasound follow up & $10(9.4)$ & $57(53.8)$ & \\
\hline Fetal testing (BPP/EFW) & $28(26.4)$ & $3(2.8)$ & \\
\hline Placenta & $2(1.9)$ & $2(1.9)$ & \\
\hline Procedure and postprocedure & $5(4.7)$ & $1(0.9)$ & $\mathbf{0 . 0 0 5}$ \\
\hline Polyhydramnios ${ }^{a}$ & $11(10.4)$ & $3(2.8)$ & $\mathbf{0 . 0 0 5}$ \\
\hline Oligohydramnios ${ }^{a}$ & $15(14.2)$ & \\
\hline
\end{tabular}

Abbreviations: BPP, biophysical profile; CAS, chorioamniotic separation; EFW, estimated fetal weight.

Notes: Data are median (interquartile range) or $n(\%)$ unless otherwise specified.

Bold indicates statistical significance at the $p \leq 0.05$ level.

aPolyhydramnios or oligohydramnios at the time of ultrasound (diagnosis of CAS for the case group or matching ultrasound without CAS for the control group). At our institution, the amniotic fluid volume is assessed by a qualitative method. This assessment is a subjective interpretation by the sonographer's clinical experience without sonographic measurements.

membranes was 4.2 weeks (IQR, 1.7-10.9 weeks) and from diagnosis to delivery was 15.6 weeks (IQR, 5.2-20.1 weeks). Those pregnancies diagnosed after 24 weeks had a median time from diagnosis to rupture of membranes of 1.6 weeks (IQR, 0.9-5.0 weeks) and from diagnosis to delivery of 0.6 weeks (IQR, 0.03-1.5 weeks).

- Table 4 shows the predictors of preterm delivery in our multivariate model. CAS is an independent risk factor for preterm delivery (adjusted odds ratio [aOR], 5.6; 95\% confidence interval $[\mathrm{CI}], 2.5-12.2)$. The magnitude of the aOR is similar to that of a history of spontaneous preterm birth (aOR, $6.9 ; 95 \% \mathrm{CI}, 2.2-21.7$ ).
In addition, we performed a sensitivity analysis where we only included a subgroup of patients who did not have risk factors for preterm delivery and the association between CAS and preterm delivery was significant, consistent with the primary analysis. In this subanalysis of a lower risk population, the rate of preterm delivery in the cases was $34.8 \%(8 / 23)$ compared with the rate of $8.2 \%(5 / 61)$ in the controls (aOR, 6.0, 95\% CI, 1.7-20.9; $p=0.005)$.

The details regarding the cases of poor pregnancy outcomes and aneuploidy for both spontaneous and iatrogenic cases of CAS are shown in Table S1 (-Supplementary Material available in the online version only). 
Table 2 Perinatal outcomes of the control and case groups

\begin{tabular}{|c|c|c|c|}
\hline Pregnancy outcomes & $\begin{array}{l}\text { Case group } \\
n=94\end{array}$ & $\begin{array}{l}\text { Control group } \\
n=105\end{array}$ & $p$ Value \\
\hline \multicolumn{4}{|l|}{ Preterm birth (wk) } \\
\hline$<24$ & $4(4.3)$ & $1(1.0)$ & 0.19 \\
\hline$<28$ & $10(10.7)$ & $2(1.9)$ & 0.01 \\
\hline$<34$ & $30(31.9)$ & $5(4.8)$ & $<0.0001$ \\
\hline$<37$ & $54(57.5)$ & $18(17.1)$ & $<0.0001$ \\
\hline Gestational age at delivery (wk) & $36(32.9-38.6)$ & $39(37.9-39.7)$ & $<0.0001$ \\
\hline Spontaneous preterm birth & $37(39.4)$ & $10(9.5)$ & $<0.0001$ \\
\hline Preterm rupture of membranes & $32(34.0)$ & $5(4.8)$ & $<0.0001$ \\
\hline Placental abruption & $11(11.7)$ & $1(1.0)$ & 0.002 \\
\hline Chorioamnionitis & $13(13.8)$ & $3(2.8)$ & 0.007 \\
\hline Birth weight (g) & $2,665(1,920-3,075)$ & $3,066(2,784-3,505)$ & $<0.0001$ \\
\hline Intrauterine growth restriction & $8(8.5)$ & $11(10.6)$ & 0.64 \\
\hline 5 min Apgar $<7$ & $18(19.2)$ & $3(2.9)$ & $<0.0001$ \\
\hline NICU Admission & $51(54.3)$ & $30(28.9)$ & 0.0003 \\
\hline Stillbirth & $3(3.2)$ & 0 & 0.10 \\
\hline Neonatal demise & $4(4.3)$ & $1(0.6)$ & 0.19 \\
\hline Mode of delivery & & & 0.23 \\
\hline Vaginal & $48(51.1)$ & 64 (60.9) & \\
\hline Cesarean & 45 (47.9) & $41(39.0)$ & \\
\hline
\end{tabular}

Abbreviation: NICU, neonatal intensive care unit.

Notes: Data are median (interquartile range) or $n$ (\%) unless otherwise specified.

Bold indicates statistical significance at the $p \leq 0.05$ level.

Table 3 Cases of chorioamniotic separation: perinatal outcomes stratified by type of chorioamniotic separation (spontaneous vs. iatrogenic), time of diagnosis (before vs. after 24 wk gestation) and by degree of severity (mild vs. moderate/severe)

\begin{tabular}{|c|c|c|c|c|c|c|c|c|c|}
\hline \multirow{2}{*}{$\begin{array}{l}\text { Perinatal } \\
\text { outcomes }\end{array}$} & \multicolumn{3}{|l|}{ Type of CAS } & \multicolumn{3}{|c|}{ Time of diagnosis } & \multicolumn{3}{|c|}{ Degree of severity } \\
\hline & $\begin{array}{l}\text { Spontaneous } \\
n=64\end{array}$ & $\begin{array}{l}\text { latrogenic } \\
n=30\end{array}$ & $\begin{array}{l}p \\
\text { Value }\end{array}$ & $\begin{array}{l}<24 \text { wk } \\
n=31\end{array}$ & $\begin{array}{l}>24 w k \\
n=63\end{array}$ & $\begin{array}{l}p \\
\text { Value }\end{array}$ & $\begin{array}{l}\text { Mild } \\
\mathrm{n}=55\end{array}$ & $\begin{array}{l}\text { Moderate/severe } \\
n=39\end{array}$ & $\begin{array}{l}p \\
\text { Value }\end{array}$ \\
\hline \multicolumn{10}{|c|}{ Preterm delivery (wk) } \\
\hline$<24$ & $3(4.7)$ & $1(3.3)$ & 1.00 & 4 (12.9) & 0 & & $1(1.8)$ & $3(7.7)$ & 0.30 \\
\hline$<28$ & 7 (10.9) & $3(10.0)$ & 1.00 & $8(25.8)$ & $2(3.2)$ & 0.002 & $4(7.3)$ & $6(15.4)$ & 0.31 \\
\hline$<34$ & $21(32.8)$ & $9(30.0)$ & 0.78 & $12(38.7)$ & $18(28.6)$ & 0.32 & $16(29.1)$ & $14(35.9)$ & 0.49 \\
\hline$<37$ & $33(51.6)$ & $21(70)$ & 0.09 & $17(54.8)$ & $37(58.7)$ & 0.72 & $31(56.4)$ & $23(59.0)$ & 0.80 \\
\hline SPTD & $26(40.6)$ & $11(36.7)$ & 0.71 & $11(35.4)$ & $26(41.3)$ & 0.59 & $23(41.8)$ & $14(35.9)$ & 0.56 \\
\hline PPROM & $11(36.7)$ & $21(32.8)$ & 0.71 & $9(29.0)$ & $23(36.5)$ & 0.47 & $20(36.4)$ & $12(30.8)$ & 0.57 \\
\hline IUGR & $8(12.5)$ & 0 & 0.05 & $3(9.7)$ & $5(7.9)$ & 1.00 & $5(9.1)$ & $3(7.7)$ & 1.00 \\
\hline Stillbirth & $2(3.1)$ & $1(3.13)$ & 1.00 & $3(9.7)$ & 0 & 0.03 & 0 & $3(7.7)$ & 0.06 \\
\hline Demise & $2(3.1)$ & $2(6.7)$ & 0.60 & $3(9.7)$ & $1(1.6)$ & 0.10 & $1(1.8)$ & $3(7.7)$ & 0.31 \\
\hline
\end{tabular}

Abbreviations: CAS, chorioamniotic separation; IUGR, intrauterine growth restriction; PPROM, preterm premature rupture of membranes; SPTB, spontaneous preterm delivery.

Note: Bold indicates statistical significance at the $p \leq 0.05$ level. 
Table 4 Predictors of preterm delivery, multivariate model

\begin{tabular}{|l|l|l|}
\hline \multicolumn{3}{|l|}{ Multivariate analysis } \\
\hline Variables & aOR (95\% Cl) & $p$ Value \\
\hline Chorioamniotic separation & $5.6(2.5-12.2)$ & $<\mathbf{0 . 0 0 0 1}$ \\
\hline Advanced maternal age & $0.6(0.3-1.4)$ & 0.25 \\
\hline White race & $1.7(0.8-3.6)$ & 0.21 \\
\hline In vitro fertilization & $2.3(0.7-7.4)$ & 0.16 \\
\hline $\begin{array}{l}\text { History of spontaneous } \\
\text { preterm delivery }\end{array}$ & $6.9(2.2-21.7)$ & $\mathbf{0 . 0 0 0 9}$ \\
\hline Invasive procedure & $2.4(1.1-5.3)$ & $\mathbf{0 . 0 3}$ \\
\hline Vaginal bleeding & $3.4(1.2-9.3)$ & $\mathbf{0 . 0 2}$ \\
\hline Subchorionic hematoma & $0.8(0.2-2.7)$ & 0.71 \\
\hline $\begin{array}{l}\text { Polyhydramnios/ } \\
\text { oligohydramnios }\end{array}$ & $3.8(1.4-10.5)$ & $\mathbf{0 . 0 1}$ \\
\hline Abnormal karyotype & $2.5(0.2-37.5)$ & 0.50 \\
\hline
\end{tabular}

Abbreviations: aOR, adjusted odds ratio; $\mathrm{Cl}$, confidence interval. Note: Bold indicates statistical significance at the $p \leq 0.05$ level.

\section{Discussion}

We found that CAS is highly associated with a greater than threefold increased risk of preterm delivery ( 57.45 vs. $17.14 \%$, $p<0.0001$ ). Even more striking is the greater than fourfold increased risk of spontaneous preterm delivery (39.4 vs. 9.5\%, $p<0.0001$ ). The higher rate of preterm delivery was independent of other potential confounders, such as maternal age, parity, obstetric history, and vaginal bleeding/subchorionic hematoma (aOR, 5.6; 95\% Cl, 2.5-12.2). This is the largest study of CAS at a single academic institution, including 74 cases of spontaneous CAS and 32 cases of iatrogenic CAS. There has been no published literature comparing outcomes of CAS based on whether it occurred spontaneously or after an invasive intrauterine procedure, gestational age at diagnosis, or degree of severity.

Similarly to prior studies by Levine et al and Bromley et al, our study shows an association between CAS and a higher rate of preterm delivery. ${ }^{2,3}$ As postulated by Levine et al, the association between CAS and preterm birth and preterm rupture of membranes could be explained by a weakened or altered mechanical function of the chorioamniotic membrane. $^{3}$ Our results showed that not only the rate of preterm delivery, but also the overall rate of preterm rupture of membranes and the overall rate of spontaneous preterm delivery were higher for patients with CAS.

The preterm delivery rate for our control group was $17 \%$, which is higher than the baseline preterm delivery rate in the United States of $11.4 \% .^{9}$ This higher preterm birth rate in the control group could be explained by the volume of high-risk patients that are seen at our institution. We gathered our controls by looking at those patients who underwent obstetric ultrasound without a diagnosis of CAS; thus, potentially selecting for higher risk patients as we only included those undergoing ultrasound examinations. However, this was the most appropriate comparison group as the only way to diagnosing CAS is by ultrasound.
The indication for ultrasound examination was significantly different between the two groups with a higher frequency of risk factors associated with preterm delivery in the CAS group ( - Table $\mathbf{1}$ ). Given this difference and the higher than expected preterm delivery rate in our control group, we performed a sensitivity analysis and excluded all patients with risk factors for preterm delivery from both the CAS and the control groups. In this subanalysis, the rate of preterm delivery in the control group was $8.2 \%$ (closer to the national baseline). Furthermore, after exclusion of the higher risk patients in both groups, the rate of preterm birth was still significantly higher in the CAS group than in the control group (34.8 vs. $8.2 \% ; p=0.005$ ).

Prior literature has shown an association between CAS and aneuploidy, particularly trisomy $21 .{ }^{2}$ Even though the rate of aneuploidy was higher in the cases of CAS, our study showed that this rate was not significantly different between the two cohorts. It is important to note that not all patients in the study had a known karyotype ( $41.5 \%$ of the cases and $18.9 \%$ of the controls had diagnostic genetic testing). Aneuploidy is a rare outcome and our study was not powered to detect this difference. In Bromley's case series, the three cases of trisomy 21 had other sonographic findings suggestive of aneuploidy. ${ }^{2}$ At our institution, all cases of fetal aneuploidy with CAS $(n=6)$ had other abnormal findings on ultrasound such as cystic hygroma, choroid plexus cysts, and heart abnormalities. There were no cases of an abnormal karyotype with an isolated finding of CAS (Table S1, - Supplementary Material available in the online version only). Given our small numbers of fetal aneuploidy, our results should not be extrapolated to consider that a normal ultrasound precludes fetal aneuploidy.

In terms of rates of stillbirth and neonatal demise, there were no significant differences between the two cohorts. Stillbirths and neonatal demises were not grouped together as perinatal deaths given that the risk factors associated with these adverse outcomes were most likely not similar. The neonatal demises $(n=4)$ of our CAS group included three cases of previable preterm rupture of membranes and a case of an intrapartum demise after a nonreassuring fetal heart tracing following an amnioreduction for polyhydramnios for a fetus with a normal karyotype and pyloric atresia. There were no cases of intrauterine fetal demise in patients who were diagnosed with a CAS after 24 weeks; however, the rate of stillbirth was significantly higher for those cases that were diagnosed before 24 weeks $(n=3) \quad(9.7$ vs. $0 \%$, $p=0.03 ;-$ Table 3). Even though it was not significant, there was a trend toward more cases of stillbirth in the moderate/ severe group than in the mild group of CAS (7.7 vs. $0 \%$, $p=0.06$ ). Levine et al had found that complete CAS was associated with morbidity and mortality. ${ }^{3}$ She postulated that the fetal deaths could be explained by the rupture of the amnion and the subsequent development of amniotic bands that wrap around the umbilical cord causing constriction. ${ }^{3}$ Table S1 (- Supplementary Material available in the online version only) shows the placental pathology and postmortem examination reports of our cases of stillbirth (including clinical comments made by the respective pathologists). The causes of death were not generalizable to umbilical 
cord constriction from amniotic band syndrome explicitly; however, this is a very difficult diagnosis to make in the setting of other variables that can contribute to stillbirths such as oligohydramnios and a known CAS.

Our study showed no significant differences in pregnancy outcomes between spontaneous and iatrogenic cases of CAS. However, there was a trend toward more cases of intrauterine growth restriction with spontaneous CAS than with iatrogenic CAS ( 12.5 vs. $0 \%, p=0.05$ ). It is important to note that $20.3 \%$ $(15 / 74)$ of the spontaneous cases had an amniocentesis procedure after the diagnosis was made and this procedure could have potentially altered the pregnancy course or change the pregnancy complication rate.

Strengths of our study include a large number of cases at one single center in comparison with what has been previously published. In addition, our selection of a comparison group (those who underwent an ultrasound at the same institution without a diagnosis of CAS) allows for a more accurate assessment of risk, as opposed to a comparison to a national average only. Furthermore, our study was adequately powered to detect a preterm delivery rate difference between our two cohorts. Our study was performed over 14 years, a period of time in which not only the quality of diagnostic ultrasound imaging has changed but also the management of pregnancy complications such as preterm labor. However, cases and controls were matched for date/year of ultrasound, controlling for this bias.

One of the main limitations of our study is that it is retrospective; thus, the true frequency, onset, and persistence of CAS remain unknown. We cannot be certain whether the patients in the control group that delivered preterm had a CAS that was simply not recognized or not reported prenatally. Another limitation is our study's reliance on chart review. There were nine cases (8.5\%) with missing pregnancy outcomes and it is possible that these cases were at a lower risk for preterm birth, as they delivered at an outside institution. However, given the large increased risk of preterm birth, it is unlikely that including these outcomes, even if healthy term deliveries, would have appreciably changed our main findings. At last, there is limited generalizability of our results given that our research was performed at a single institution.

Our findings make a significant contribution to the published literature because we have a large number of cases of spontaneous CAS and we make a distinction between spon- taneous and iatrogenic cases, gestational age at the time of diagnosis, and degree of severity. Our results show that CAS is significantly associated with preterm delivery and if diagnosed before 24 weeks, the rate of stillbirth is significantly higher. Given these findings, routine reporting of CAS should be considered and close clinical surveillance is warranted in the presence of this diagnosis.

Note

The study is original research that was performed at the Brigham and Women's Hospital, Boston, MA. The authors report no conflict of interest and there was no financial support provided for this project.

\section{References}

1 Kim YN, Jeong DH, Jeong SJ, Sung MS, Kang MS, Kim KT. Complete chorioamniotic membrane separation with fetal restrictive dermopathy in two consecutive pregnancies. Prenat Diagn 2007;27(4): 352-355

2 Bromley B, Shipp TD, Benacerraf BR. Amnion-chorion separation after 17 weeks' gestation. Obstet Gynecol 1999;94(6):1024-1026

3 Levine D, Callen PW, Pender SG, et al. Chorioamniotic separation after second-trimester genetic amniocentesis: importance and frequency. Radiology 1998;209(1):175-181

4 Benacerraf BR, Frigoletto FD Jr. Sonographic observation of amniotic rupture without amniotic band syndrome. J Ultrasound Med 1992;11(2):109-111

5 Graf JL, Bealer JF, Gibbs DL, Adzick NS, Harrison MR. Chorioamniotic membrane separation: a potentially lethal finding. Fetal Diagn Ther 1997;12(2):81-84

6 Sydorak RM, Hirose S, Sandberg PL, et al. Chorioamniotic membrane separation following fetal surgery. J Perinatol 2002;22(5): 407-410

7 Ulm B, Ulm MR, Bernaschek G. Unfused amnion and chorion after 14 weeks of gestation: associated fetal structural and chromosomal abnormalities. Ultrasound Obstet Gynecol 1999;13(6):392-395

8 Lewi L, Hanssens M, Spitz B, Deprest J. Complete chorioamniotic membrane separation. Case report and review of the literature. Fetal Diagn Ther 2004;19(1):78-82

9 Martin JA, Hamilton BE, Osterman MJ, Curtin SC, Matthews TJ. Births: final data for 2013. Natl Vital Stat Rep 2015;64(1):1-65

10 Harris PA, Taylor R, Thielke R, Payne J, Gonzalez N, Conde JG. Research electronic data capture (REDCap)-a metadata-driven methodology and workflow process for providing translational research informatics support. J Biomed Inform 2009;42(2): 377-381 